\title{
Dieta Intermitente Atenua a Remodelação Cardíaca Causada pelo Exercício Físico
}

\author{
Intermittent Fasting Attenuates Exercise Training-Induced Cardiac Remodeling
}

Priscilla Gois Basilio, ${ }^{1}$ Ana Priscila Cayres de Oliveira, ${ }^{1}$ Ana Carolini Ferreira de Castro, ${ }^{1}$ Marianna Rabelo de

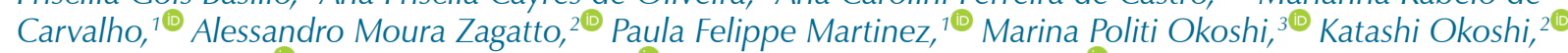
Gabriel Elias Ota, ${ }^{10}$ Filipe Abdalla dos Reis, ${ }^{40}$ Silvio Assis de Oliveira-Junior ${ }^{10}$

Laboratório de Estudo do Músculo Estriado (LEME/INISA), Universidade Federal de Mato Grosso do Sul, ${ }^{1}$ Campo Grande, MS - Brasil Departamento de Educação Física, Laboratório de Fisiologia e Desempenho Esportivo (LAFIDE), Faculdade de Ciências - Universidade Estadual Paulista (UNESP), ${ }^{2}$ Bauru, SP - Brasil

Faculdade de Medicina de Botucatu - Departamento de Clínica Médica - Universidade Estadual Paulista (UNESP), ${ }^{3}$ Botucatu, SP - Brasil Centro Universitário Anhanguera de Campo Grande, ${ }^{4}$ Campo Grande, MS - Brasil

\section{Resumo}

Fundamento: A influência de intervenções não farmacológicas como restrição calórica e exercício físico sobre a saúde e prevenção de enfermidades cardíacas tem sido documentada em estudos clínicos e experimentais.

Objetivo: Analisar a influência da combinação entre dieta intermitente e exercício físico sobre a capacidade funcional, metabolismo glicêmico e remodelação cardíaca.

Métodos: Foram utilizados 60 ratos Wistar machos distribuídos em quatro grupos: Controle (C), Exercício Físico (EF), Dieta Intermitente (DI) e Exercício Físico e Dieta Intermitente (EDI). Durante 12 semanas, enquanto C e EF foram tratados diariamente com dieta comercial padrão ad libitum, DI e EDI receberam dieta similar em dias alternados com dias de jejum. Os grupos EF e EDI foram submetidos a protocolo de corrida em esteira rolante. Posteriormente, foram analisadas capacidade funcional, comportamento nutricional e metabolismo glicêmico. Além da morfologia do coração, a expressão proteica das proteínas extracellular signal-regulated kinase (ERK) e c-Jun N-terminal kinase (JNK) no coração foi avaliada por Western-blot. A análise dos resultados foi feita por meio de Two-Way ANOVA e teste de Student-Newman-Keuls. O nível de significância considerado foi de $5 \%$.

Resultados: O exercício físico aumentou a capacidade funcional nos grupos EF e EDI, e acarretou fibrose cardíaca. A combinação entre dieta intermitente e exercício físico resultou em menor área sob a curva de glicemia e menores medidas de área e interstício cardíaco no EDI em relação ao EF. A expressão de proteínas ERK e JNK foi similar entre os grupos $(p>0,05)$.

Conclusões: Dieta intermitente se associa com melhor tolerância glicêmica e atenua o processo de remodelação cardíaca decorrente do exercício físico. (Arq Bras Cardiol. 2020; 115(2):184-193)

Palavras-chave: Dieta Saudável, Restrição Calórica, Exercício Físico, Corrida, Remodelação Ventricular, Índice de Glicemia, Promoção da Saúde.

\footnotetext{
Abstract

Background: The effects of non-pharmacological interventions such as calorie restriction and exercise training on health and prevention of cardiovascular diseases have been investigated in clinical and experimental studies.

Objective: To analyze the influence of intermittent fasting and exercise training on functional fitness, glycemia and cardiac remodeling.

Methods: Wistar rats $(n=60)$ were randomly divided into four groups: control, exercise training (ET), intermittent fasting (IF) and exercise training plus intermittent fasting (ETI). Over 12 weeks, control and ET animals were fed daily a standard commercial diet ad libitum, while IF and ETI animals were fed every other day. In addition, the ET and ETI groups were submitted to a running protocol on a treadmill. After this period, functional fitness, nutritional parameters and blood glucose levels were analyzed. In addition to heart morphology, myocardial protein expression of extracellular signal-regulated kinase (ERK) and c-Jun N-terminal kinase (JNK) was assessed by Western-blot. The results were analyzed using two-way ANOVA and Student-Newman-Keuls test. The level of significance considered was 5\%.
}

Correspondência: Silvio Assis de Oliveira-Junior •

Universidade Federal de Mato Grosso do Sul (UFMS), Instituto Integrado de Saúde, Laboratório de Estudo do Músculo Estriado (LEME/INISA) - Av. Costa e Silva, s/n. CEP 79070-900, Cidade Universitária, Campo Grande, MS - Brasil

E-mail: oliveirajr.ufms@gmail.com, silvio.oliveira-jr@ufms.br

Artigo recebido em 29/05/2019, revisado em 14/08/2019, aceito em 14/08/2019

DOI: https://doi.org/10.36660/abc.20190349 
Results: Exercise training increased functional fitness in the ET and ETI groups and promoted cardiac fibrosis. The combination of intermittent fasting and exercise training resulted in a smaller area under the blood glucose curve and reduced cardiomyocyte cross-sectional area and interstitial collagen fraction in the ETI group compared to ET. ERK and JNK expression levels were similar among groups ( $\mathrm{p}>0.05$ ).

Conclusion: Intermittent fasting is associated with improved glucose tolerance and attenuates cardiac remodeling induced by exercise training. (Arq Bras Cardiol. 2020; 115(2):184-193)

Keywords: Diet; Calorie Restriction; Exercise; Running; Ventricular Remodeling; Glucose; Health Promotion.

Full texts in English - http://www.arquivosonline.com.br

\section{Introdução}

Classicamente, a restrição calórica é popularmente adotada como intervenção para melhorar a saúde, visando a promoção de benefícios funcionais ao organismo e maior longevidade. ${ }^{1-4}$ Entretanto, estudos experimentais têm mostrado respostas controversas no aspecto cardiovascular, pois a restrição calórica se mostrou associada com disfunção contrátil e danos morfológicos no miocárdio..$^{5-8}$ Alguns pesquisadores constataram que a restrição calórica resultou em lesões de ultraestrutura miofibrilar e mudanças no trânsito intracelular de cálcio, relacionadas com distúrbios do sistema $\beta$-adrenérgico, contribuindo, assim, para disfunção contrátil do miocárdio. ${ }^{5,7,9}$ As modificações morfológicas envolveram também dilatação de câmaras ventriculares, degeneração dos cardiomiócitos, fibrose intersticial e edema mitocondrial. ${ }^{10-12}$

Além disso, após 12 semanas de experimento, o modelo intermitente de restrição calórica esteve pouco associado com danos morfológicos e não promoveu disfunção miocárdica, em comparação à restrição calórica de $50 \% .{ }^{11} \mathrm{Na}$ restrição calórica intermitente, o alimento é disponibilizado ad libitum em intervalos alternados com períodos de jejum, cada qual com duração de 12 a 24 horas., ${ }^{3,4}$ São escassos os estudos sobre os efeitos desse tipo de intervenção sobre o coração. No aspecto molecular, inclusive, a participação de proteínas cinases ativadas do mitógeno (MAPK), importantes agentes do processo de remodelação cardíaca, ${ }^{13}$ não foi ainda estudada em modelos de restrição calórica. As MAPKs incluem três subtipos principais, extracellular signal-regulated (ERK), c-Jun N-terminal (JNK) e p38 (p38K), que regulam a transcrição gênica de diversos mensageiros envolvidos na sobrevida, apoptose, diferenciação celular e remodelação cardíaca. ${ }^{13,14}$

Por sua vez, a prática regular de exercício físico é amplamente difundida como medida de promoção de saúde e prevenção de diferentes condições cardiovasculares ${ }^{15,16}$ Entretanto, diferentes estudos experimentais mostraram resultados controversos, evidenciando que o exercício físico não afetou e, inclusive, reduziu o desempenho miocárdico. ${ }^{8,9,17-19}$ Além disso, não foram encontradas evidências em relação à influência de protocolos de exercício físico em esteira rolante sobre o processo de remodelação cardíaca na restrição calórica intermitente.

Portanto, o objetivo do presente estudo foi analisar a influência da combinação entre restrição calórica intermitente e prática de exercício físico sobre o desempenho físico ao esforço e indicadores morfológicos e moleculares de remodelação miocárdica. Como hipótese inicial do presente estudo, admite-se que o exercício físico amplia o desempenho físico e atenua a remodelação miocárdica decorrente da restrição calórica intermitente.

\section{Métodos}

O projeto científico foi analisado e aprovado pelo Comitê de Ética no Uso de Animais (CEUA/UFMS; Protocolo 615/2014), estando em conformidade com os regimentos do Colégio Brasileiro de Experimentação Animal (COBEA).

\section{Animais e Protocolo Experimental}

Foram utilizados 60 ratos da linhagem Wistar (Rattus novergicus albinus), machos, com 60 dias de idade, procedentes do Biotério Central da Universidade Federal de Mato Grosso do Sul (UFMS). A definição do tamanho amostral baseou-se em estudo prévio ${ }^{16}$ e considerou a probabilidade de recusa ao exercício e/ou instinto de fuga durante o teste de esforço. ${ }^{20,21}$ Utilizando-se de amostragem casual simples, os animais foram distribuídos em quatro grupos: Controle (C), Dieta Intermitente (DI), Exercício Físico (EF) e Exercício Físico e Dieta Intermitente (EDI). Enquanto CT e EF foram tratados diariamente com ração ad libitum (Nuvilab ${ }^{\circledR}$, Brasil), os grupos DI e EDI receberam tratamento similar, administrado em dias alternados com dias de jejum.

Além do suporte nutricional, os animais dos grupos EF e EDI foram submetidos a um protocolo de corrida em esteira rolante (Tabela 1) elaborado de acordo com estudos prévios. ${ }^{16,20}$ Foram realizadas cinco sessões semanais de exercício físico e o período experimental perdurou por 12 semanas. Os animais foram mantidos em gaiolas coletivas com duas a três unidades por caixa, sob temperatura ambiente de $22 \pm 2{ }^{\circ} \mathrm{C}$, umidade de $55 \pm 5 \%$, ciclos de iluminação claro/ escuro de 12 horas e água sob livre acesso.

\section{Teste de Esforço}

Para analisar a capacidade funcional, ao término do experimento, realizou-se teste incremental de estágios múltiplos conforme estudos prévios. ${ }^{21,22} \mathrm{O}$ teste foi iniciado com aquecimento de 5 minutos à velocidade de $5 \mathrm{~m} / \mathrm{min}$. Após 1 min de intervalo, cada animal foi submetido a um esforço progressivo, com velocidade inicial de $6 \mathrm{~m} / \mathrm{min}$,

Tabela 1 - Protocolo de exercício físico em esteira rolante, segundo período, velocidade média e duração das sessões

\begin{tabular}{lcc}
\hline Período & Velocidade $(\mathrm{m} / \mathrm{min})$ & Duração $(\mathrm{min})$ \\
\hline $1^{\mathrm{a}}-3^{\mathrm{a}}$ semana & 10 & $40-60$ \\
$4^{\mathrm{a}}-6^{\mathrm{a}}$ semana & 15 & 40 \\
$7^{\mathrm{a}}-9^{\mathrm{a}}$ semana & 18 & 35 \\
$10^{\mathrm{a}}-12^{\mathrm{a}}$ semana & 19 & $15-25$ \\
\hline
\end{tabular}


seguido por incrementos de $3 \mathrm{~m} / \mathrm{min}$, que perdurou por 3 minutos. O protocolo foi finalizado quando o animal atingisse a exaustão ou quando a coordenação entre as passadas se apresentasse dificultada. ${ }^{21}$

Para avaliar a resposta de lactato, $25 \mu \mathrm{l}$ de sangue foram coletadas da cauda do animal em repouso e após cada estágio de esforço. O sangue coletado foi imediatamente armazenado em tubo Eppendorf contendo $50 \mu \mathrm{l}$ de fluoreto de sódio (NaF) a $1 \%$. As amostras de sangue foram refrigeradas após a coleta e, em seguida, mantidas em freezer $\left(-20{ }^{\circ} \mathrm{C}\right)$ até a análise, que foi realizada em analisador eletroquímico YSI 150 Sport (Yellow Springs Instruments ${ }^{\circledR}$, Ohio, EUA) com erro-padrão da medida de $\pm 2 \%$.

Os resultados foram dados em $\mathrm{mmol} / \mathrm{l}$ e a determinação do limiar anaeróbico de lactato (LL) foi realizada por plotagem gráfica do comportamento das concentrações durante o teste. O LL foi determinado pelo momento de quebra da linearidade em função do aumento de carga, obtido mediante inspeção visual. A capacidade funcional foi avaliada pela velocidade no limiar de lactato (VLL), distância percorrida, concentração de lactato sanguíneo no limiar de lactato (LacLL) e no momento da exaustão (LacE), determinados durante o teste de esforço. Além disso, para melhor detalhar a cinética de lactato, considerou-se também a variação relativa (\%) de níveis de lactato, obtida a partir das medidas de lactato LacLL e LacE.

\section{Caracterização Metabólica}

Para análise do metabolismo glicêmico, os animais foram submetidos a jejum por 8-12 horas e amostras sanguíneas provenientes da artéria caudal foram utilizadas para a dosagem de glicose na condição basal. A seguir, realizouse administração intraperitoneal de glicose a 20\% (Glicose Monohidratada, Merck, São Paulo, Brasil), em dosagem equivalente a $2 \mathrm{~g} / \mathrm{kg}$. Os níveis glicêmicos foram então avaliados após 15, 30, 60, 90, 120 e 180 minutos. ${ }^{7,10}$ Para tanto, utilizou-se do glicosímetro ACCU-CHEK GO KIT (Roche Diagnostic Brazil Ltda, SP, Brasil). 23,24

\section{Caracterização Nutricional}

A caracterização nutricional envolveu ingestão alimentar (IA), ingestão calórica (IC) e eficiência energética. A IA foi avaliada diariamente e a IC foi calculada pela seguinte fórmula: IA $\times$ (valor calórico da dieta). ${ }^{23}$ A massa corporal foi mensurada semanalmente, utilizando-se uma balança digital. A variação ponderal foi obtida a partir da diferença entre os valores de massa corporal inicial e final, segundo momento de análise. Para analisar a capacidade de conversão da energia ingerida em massa corporal, considerou-se a eficiência alimentar (EA), obtida a partir da relação entre variação ponderal total (g) e energia total ingerida (kcal). ${ }^{23,24}$

Após o período experimental os animais foram mantidos em jejum por um período de oito horas, submetidos a anestesia intraperitoneal com cloridrato de cetamina (50 mg/ kg/ip; Dopalen ${ }^{\circledR}$, Sespo Indústria e Comércio Ltda - Divisão Vetbrands, Jacareí, São Paulo, Brasil) e cloridrato de xilazina $\left(10\right.$ mg/kg/ip; Anasedan ${ }^{\circledR}$, Sespo Indústria e Comércio
Ltda - Divisão Vetbrands, Jacareí, São Paulo, Brasil). Após a eutanásia por decapitação, foram executadas toracotomia e laparotomia mediana para remoção do coração e retirada de tecido adiposo branco dos compartimentos retroperitoneal e epididimal. ${ }^{24}$ Considerou-se a soma dos dois compartimentos em valores absolutos e relativos para a determinação da adiposidade corporal.

\section{Caracterização Morfológica do Coração}

Para avaliar a morfologia macroscópica do coração, foram mensuradas as massas de átrios (MA) e dos ventrículos direito (MVD) e esquerdo (MVE) em valores absolutos e em relação à massa corporal final (MCF) e o comprimento da tíbia. Posteriormente, foram retiradas amostras do ventrículo esquerdo a partir de incisão transversal a $6 \mathrm{~mm}$ do ápice. Os fragmentos foram imersos em solução tamponada de formol a $10 \%$, na qual foram mantidos por 48 horas. Cada fragmento miocárdico foi então submetido a água corrente e mantidos sob solução de etanol a $70 \%$ por mais 48 horas. Após a etapa de fixação, os materiais foram compactados em blocos de parafina. Foram confeccionadas lâminas histológicas com secções teciduais de 4 a $7 \mu$ m de espessura, submetidas à coloração com hematoxilina-eosina (HE) e picro-sirius red (PSR). Para a análise morfométrica dos cardiomiócitos, foram consideradas medidas de área cardiomiocitária e fração intersticial de colágeno do miocárdio. ${ }^{23-25}$

Para a tomada de medidas de áreas, foram consideradas lâminas coradas em HE; para cada animal, foram amostrados pelo menos 100 cardiomiócitos. As lâminas coradas por PSR foram utilizadas somente para a quantificação do conteúdo de colágeno do meio intersticial miocárdico. Fixado o campo de imagem, os componentes do tecido cardíaco foram identificados segundo a cor realçada. Os filamentos de colágeno refletiram a cor vermelha enquanto os cardiomiócitos revelaram a coloração amarela. A fração intersticial de colágeno correspondeu à medida relativa (\%) do conteúdo de colágeno sobre toda a extensão tecidual. Um mínimo de 20 campos foi utilizado e regiões perivasculares foram desconsideradas.

Como instrumental analítico, os cortes histológicos foram projetados em aumento de 40X com o auxílio de microscópio (LEICA DM LS) acoplado a uma câmera de vídeo que projeta imagens digitais em um microcomputador IBM equipado com programa analisador de imagens Image Pro-plus (Media Cybernetics, Silver Spring, Maryland, EUA).

\section{Análise da Expressão de MAPK}

Os níveis de expressão proteica de MAPK foram determinados por meio de procedimentos de Western blot, utilizando-se anticorpos primários específicos (Santa Cruz Biotechnology Inc., CA, EUA): p-JNK (sc-6254), total JNK1/2 (sc-137019), p-ERK1/2 (sc-16982), total ERK 1 (sc-93). Os níveis de proteína obtidos foram normalizados pela expressão de GAPDH (6C5, sc-32233). Os métodos de preparação tecidual e condições de eletroforese são detalhados em estudos previamente publicados. ${ }^{24,26}$ 


\section{Análise Estatística}

Para análise estatística dos resultados, utilizou-se o software Sigma Stat. Para estudar a distribuição de dados em relação à normalidade, utilizou-se o teste de Kolmogorov-Smirnov. Os resultados paramétricos foram apresentados em média e desvio-padrão e foram analisados com emprego de análise de variância de duas vias (Two-Way ANOVA) complementada com teste de comparações de Student-Newman-Keuls. Os resultados de área celular foram distribuídos em categorias segundo intervalo de medidas, utilizando-se a fórmula de Sturges. ${ }^{27}$ Posteriormente, realizou-se análise de proporção absoluta e relativa com emprego de teste de proporções multinomiais de Goodman. ${ }^{28}$ Todas as conclusões estatísticas foram discutidas ao nível de significância de 5\%.

\section{Resultados}

$\mathrm{Na}$ 1, são mostrados os valores da distância total $(\mathrm{m})$ e velocidade final $(\mathrm{m} / \mathrm{min})$ obtidos no teste de esforço, que foi realizado no final do período experimental. Ambos os grupos exercitados, EF e EDI, apresentaram maiores valores de distância total e velocidade final, em comparação aos seus respectivos controles, $\mathrm{C}$ e DI. O efeito da dieta não foi significativo nas comparações efetuadas (Figura 1A e 1B).

Considerando-se as medidas finais de lactato, referente ao momento de exaustão (LacE), verificou-se efeito significativo $(p=0,04)$ do exercício físico (C e DI:

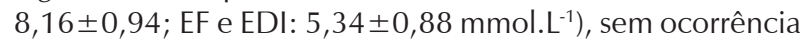
de interação fatorial. O limiar de lactato (LL) foi similar

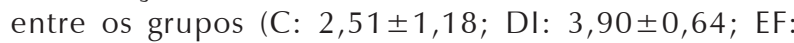
$2,70 \pm 0,23$; EDI: $\left.3,04 \pm 1,33 \mathrm{mmol} . \mathrm{L}^{-1}\right)$. A variação dos
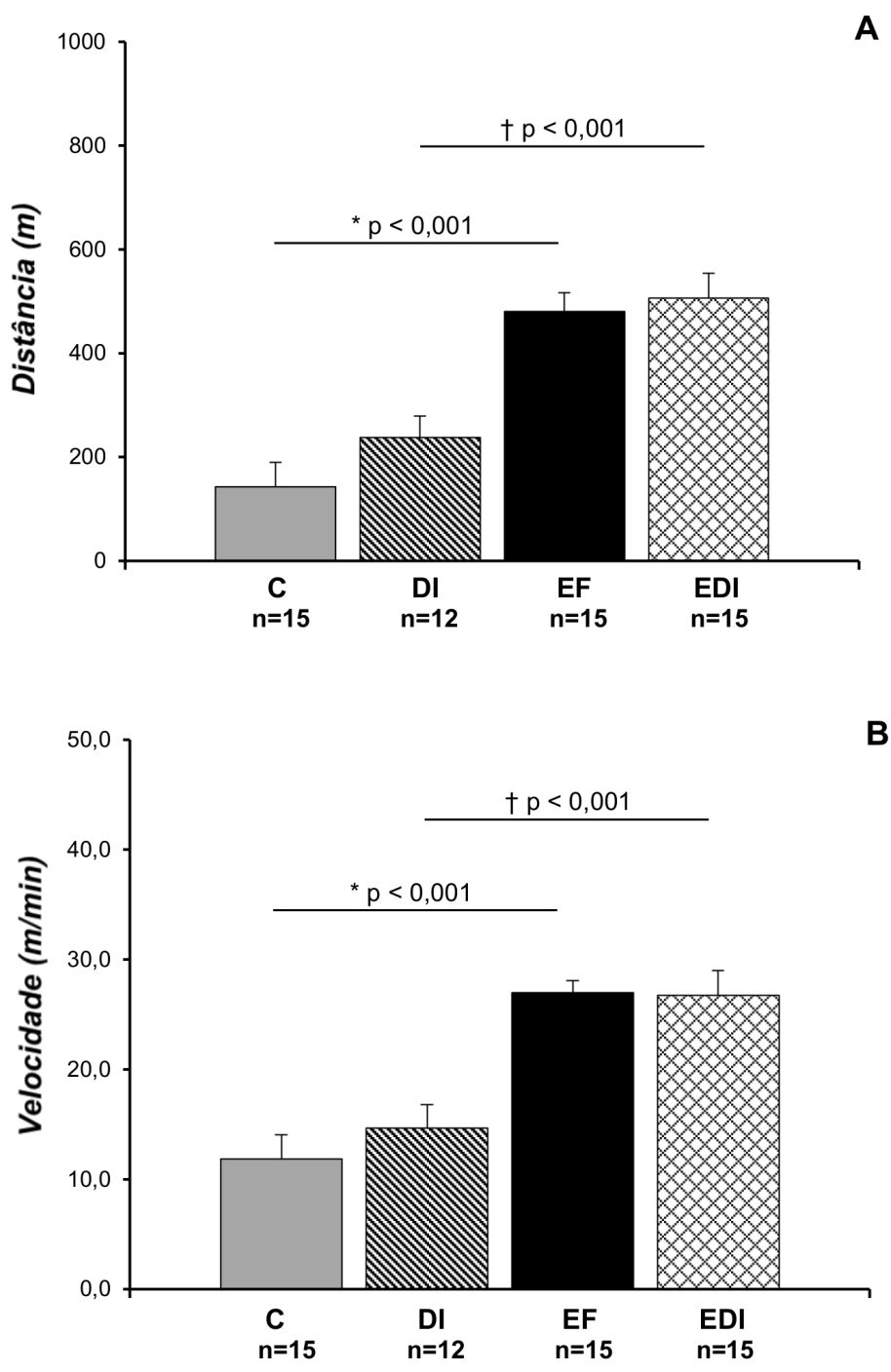

Figura 1 - Medidas de desempenho obtidas no teste de esforço, em média 土 erro-padrão; (A) Distância Percorrida; (B) Velocidade; C: grupo Controle; DI: grupo Dieta Intermitente; EF: grupo Exercício Físico; EDI: grupo Exercicio Físico e Dieta Intermitente. * $p<0,001$ vs. C; † p<0,001 vs. DI; Two-Way ANOVA e teste de StudentNewman-Keuls. 
níveis de lactato entre o ponto de inflexão e o momento final do teste foi maior $(p=0,04)$ nos grupos sedentários $(C$

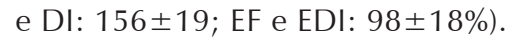

Na Figura 2, são mostrados os valores referentes à área sob a curva de tolerância glicêmica. Considerando-se o efeito isolado da dieta (Figura 2A), constatou-se que a restrição intermitente se associou com menor área de resposta glicêmica. Não foram observadas diferenças significativas em relação ao efeito isolado do exercício (Figura $2 \mathrm{C}$ ). No delineamento original (Figura 2B), o grupo EDI mostrou menor valor de área sob a curva glicêmica em relação ao EF. Não foram constatadas diferenças nas demais comparações entre grupos.
Em relação às variáveis nutricionais, as medidas de ingestão alimentar e consumo calórico foram menores nos grupos DI e EDI, quando comparados aos respectivos controles. Como fatores isolados, dieta intermitente e exercício físico resultaram em reduzida eficiência energética e menor ganho de massa corporal. Embora o grupo DI tenha mostrado menor massa corporal que o C, os valores de adiposidade não foram diferentes entre os grupos (Tabela 2).

Considerando-se os resultados de morfologia cardíaca, a dieta intermitente, per se, resultou em menores valores de massa de átrios $(0,063 \pm 0,002$ vs. 0,053 $\pm 0,002$ g; $\mathrm{p}=0,006)$ e ventrículo esquerdo, em medidas absolutas $(0,475 \pm 0,011$ vs. $0,420 \pm 0,011 \mathrm{mg} ; \mathrm{p}<0,001)$ e nas relações com o comprimento tibial. Além disso, a dieta

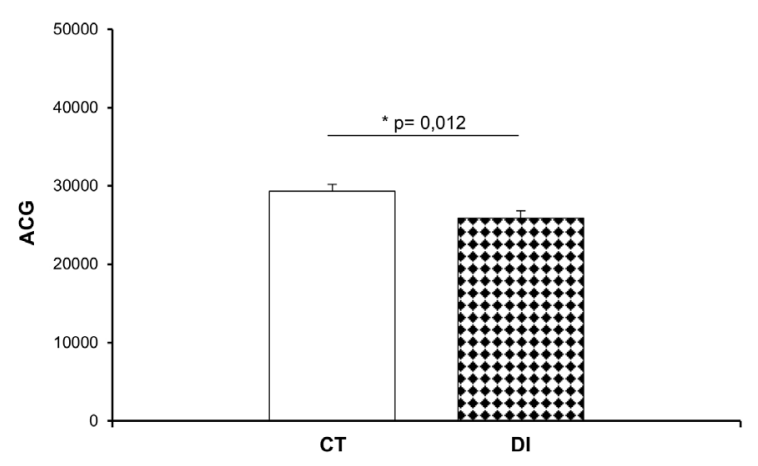

A
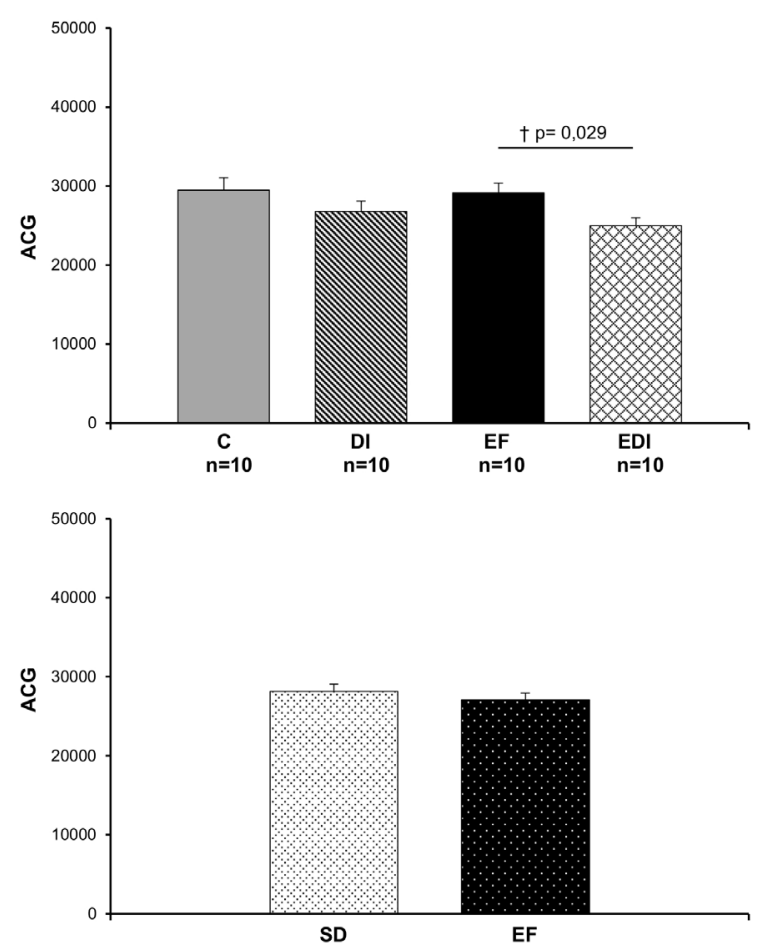

B

C

Figura 2 - Medidas de área sob a curva de tolerância glicêmica (ACG). (A) efeito isolado da dieta intermitente; CT: dieta controle ad libitum; DI: dieta intermitente; * $p<0,05$ versus $C T$. (B) efeito combinado: $C$, ratos sedentários sob dieta controle ad libitum; DI: ratos sedentários sob dieta intermitente; $E F$ : ratos exercitados sob dieta controle ad libitum; EDI: ratos exercitados sob dieta intermitente, $† p<0,05$ versus EF; (C) efeito isolado do exercício fisico; SD: grupos sedentários; EF: grupos exercitados. Two-Way ANOVA e teste de Student-Newman-Keuls 
Tabela 2 - Características nutricionais e de morfologia cardíaca segundo grupo (dieta x exercício físico)

\begin{tabular}{|c|c|c|c|c|c|c|c|c|}
\hline \multirow{2}{*}{\multicolumn{2}{|c|}{ Características }} & \multicolumn{4}{|c|}{ Grupo } & \multicolumn{3}{|c|}{ Fatores (p-valor) } \\
\hline & & $\underset{n=15}{C}$ & $\begin{array}{c}\text { DI } \\
n=15\end{array}$ & $\begin{array}{c}E F \\
n=15\end{array}$ & $\begin{array}{c}\text { EDI } \\
n=15\end{array}$ & Dieta & Condição & Interação \\
\hline \multirow{7}{*}{ 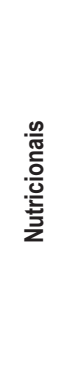 } & IA (g/dia) & $23,30 \pm 0,88$ & $17,64 \pm 1,00$ * & $22,88 \pm 0,65$ & $17,88 \pm 0,77 \dagger$ & $<0,001$ & 0,682 & 0,126 \\
\hline & IC (kcal/dia) & $84,81 \pm 3,20$ & $64,20 \pm 3,63$ * & $83,23 \pm 2,38$ & $65,10 \pm 2,80 \dagger$ & $<0,001$ & 0,665 & 0,121 \\
\hline & ICT (kcal/dia) & $7124 \pm 269$ & $5393 \pm 305^{*}$ & $6980 \pm 192$ & $5468 \pm 236 \dagger$ & $<0,001$ & 0,602 & 0,100 \\
\hline & $\mathrm{EE}(\mathrm{kcal} / \mathrm{g})$ & $0,023 \pm 0,005$ & $0,019 \pm 0,004$ * & $0,020 \pm 0,004$ & $0,017 \pm 0,005$ & 0,005 & 0,050 & 0,581 \\
\hline & $M C(g)$ & $395 \pm 46$ & $344 \pm 37$ * & $374 \pm 39$ & $349 \pm 30$ & $<0,001$ & 0,400 & 0,202 \\
\hline & VM (\%) & $71,0 \pm 17,8$ & $42,8 \pm 12,0$ * & $60,5 \pm 15,2$ & $37,4 \pm 13,6 \dagger$ & $<0,001$ & 0,043 & 0,498 \\
\hline & Adiposidade (\%) & $2,11 \pm 0,51$ & $1,91 \pm 0,77$ & $1,89 \pm 0,72$ & $1,89 \pm 0,79$ & 0,584 & 0,508 & 0,578 \\
\hline \multirow{10}{*}{ 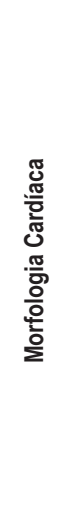 } & $M A(g)$ & $0,059 \pm 0,012$ & $0,052 \pm 0,009$ & $0,066 \pm 0,013$ & $0,055 \pm 0,015 \dagger$ & 0,006 & 0,126 & 0,422 \\
\hline & $\operatorname{MVD}(\mathrm{g})$ & $0,134 \pm 0,017$ & $0,118 \pm 0,034$ & $0,145 \pm 0,040$ & $0,130 \pm 0,020$ & 0,055 & 0,143 & 0,950 \\
\hline & MVE (g) & $0,482 \pm 0,066$ & $0,404 \pm 0,041$ * & $0,469 \pm 0,060$ & $0,436 \pm 0,059$ & $<0,001$ & 0,509 & 0,138 \\
\hline & MA/MC (mg/g) & $0,152 \pm 0,030$ & $0,152 \pm 0,026$ & $0,177 \pm 0,036$ & $0,155 \pm 0,048$ & 0,247 & 0,136 & 0,227 \\
\hline & MVD/MC (mg/g) & $0,345 \pm 0,033$ & $0,346 \pm 0,101$ & $0,380 \pm 0,076$ & $0,368 \pm 0,063$ & 0,774 & 0,135 & 0,737 \\
\hline & MVE/MC (mg/g) & $1,24 \pm 0,14$ & $1,18 \pm 0,10$ & $1,24 \pm 0,09$ & $1,23 \pm 0,14$ & 0,202 & 0,437 & 0,457 \\
\hline & $\mathrm{MA} / \mathrm{CT}(\mathrm{g} / \mathrm{cm})$ & $0,015 \pm 0,003$ & $0,013 \pm 0,002$ & $0,017 \pm 0,003$ & $0,014 \pm 0,004 \dagger$ & 0,007 & 0,227 & 0,328 \\
\hline & MVD/CT $(\mathrm{g} / \mathrm{cm})$ & $0,034 \pm 0,004$ & $0,030 \pm 0,009$ & $0,036 \pm 0,009$ & $0,032 \pm 0,004$ & 0,065 & 0,246 & 0,982 \\
\hline & MVE/CT (g/cm) & $0,121 \pm 0,015$ & $0,104 \pm 0,009$ * & $0,117 \pm 0,015$ & $0,109 \pm 0,015$ & $<0,001$ & 0,892 & 0,170 \\
\hline & Coração $(\mathrm{g})$ & $0,674 \pm 0,083$ & $0,574 \pm 0,063$ * & $0,680 \pm 0,086$ & $0,621 \pm 0,058 \dagger$ & $<0,001$ & 0,172 & 0,292 \\
\hline
\end{tabular}

IA: ingestão alimentar diária; IC: ingestão calórica diária; ICT: ingestão calórica total; EE: eficiência energética; MC: massa corporal; VM: variação relativa de massa corporal; MA: massa de átrios; MVD: massa de ventrículo direito; MVE: massa de ventrículo esquerdo; MA/MC: relação entre massa de átrios e massa corporal; MVD/MC: relação entre massa de ventrículo direito e massa corporal; MVE/MC: relação entre massa de ventrículo esquerdo e massa corporal; MA/CT: relação entre massa de átrios e comprimento da tíbia; MVD/CT: relação entre massa de ventrículo direito e comprimento da tíbia; MVE/CT: relação entre massa de ventrículo esquerdo e comprimento da tíbia. * $p<0,05$, comparado ao grupo $C ; \uparrow p<0,05$ vs. grupo EF; Two-Way ANOVA e teste de Student-Newman-Keuls.

reduziu a massa do coração $(0,677 \pm 0,013$ vs. 0,597 $\pm 0,013$ g; $\mathrm{p}<0,001$ ) (Tabela 2).

Na Figura 3, são mostradas as medidas descritivas de morfometria do miocárdio. A combinação entre dieta intermitente e exercício físico resultou em menores valores de área celular no grupo EDI, que se mostrou diferente dos

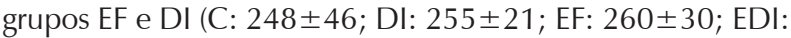
$\left.225 \pm 26 \mu \mathrm{m}^{2}\right)$. Considerando-se a distribuição categórica de cardiomiócitos, a maior parte dos resultados situou-se nas duas primeiras classes, delimitadas até $327,5 \mu \mathrm{m}^{2}$. No entanto, o grupo EDI revelou maior frequência de fibras na 1a classe de valores (até 190,1 $\mu \mathrm{m}^{2}$ ), comparado os demais grupos ( $\mathrm{p}<0,05$; Figura 3B).

Em relação ao conteúdo de colágeno, observou-se interação estatisticamente significativa entre dieta e exercício físico $(p=0,01)$. O grupo EF exibiu maior fração intersticial de colágeno em relação ao C (C: 5,32 $\pm 1,02$; DI: 5,25 $\pm 0,66$; EF:

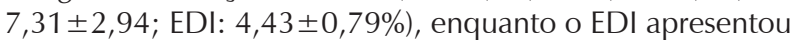
menor concentração de colágeno que o EF (Figura 4A-B).

Na Tabela 3, são apresentados os valores de expressão de proteínas MAPK ERK e JNK no miocárdio. Não foram constatadas diferenças em relação à expressão de proteínas MAPK, entre os grupos experimentais.

\section{Discussão}

Dietas restritivas vêm sendo comumente utilizadas para reduzir o risco de doenças crônicas, como obesidade, diabetes tipo 2, dislipidemia e enfermidades cardiovasculares. No presente estudo, a dieta intermitente se associou com maior consumo alimentar e energético nos dias de oferta, menor ingestão calórica total e menores medidas de massa corporal. Maior consumo alimentar decorrente da dieta intermitente pode ser explicado por alterações na sensação de saciedade. ${ }^{29,30} \mathrm{O}$ hipotálamo é um dos principais responsáveis pela homeostase corporal, exercendo diversas funções, dentre elas, a promoção de saciedade. Sabe-se que alterações no hipotálamo lateral levam à afagia (inanição), enquanto desordens no hipotálamo medial conduzem à hiperfagia (aumento do apetite). Outros estudos ${ }^{11,29}$ mostraram que a dieta intermitente acarretou maior consumo alimentar nos dias de oferta, como apresentado no presente trabalho. Da mesma forma, Dorighello et al., ${ }^{31}$ mostraram que a restrição intermitente resultou em menor ingestão calórica total, corroborando nossos achados. A vigência do exercício físico não afetou as respostas do consumo alimentar e calórico decorrentes da dieta. Buthani et al., ${ }^{32}$ em estudo com humanos, demonstraram que voluntários 


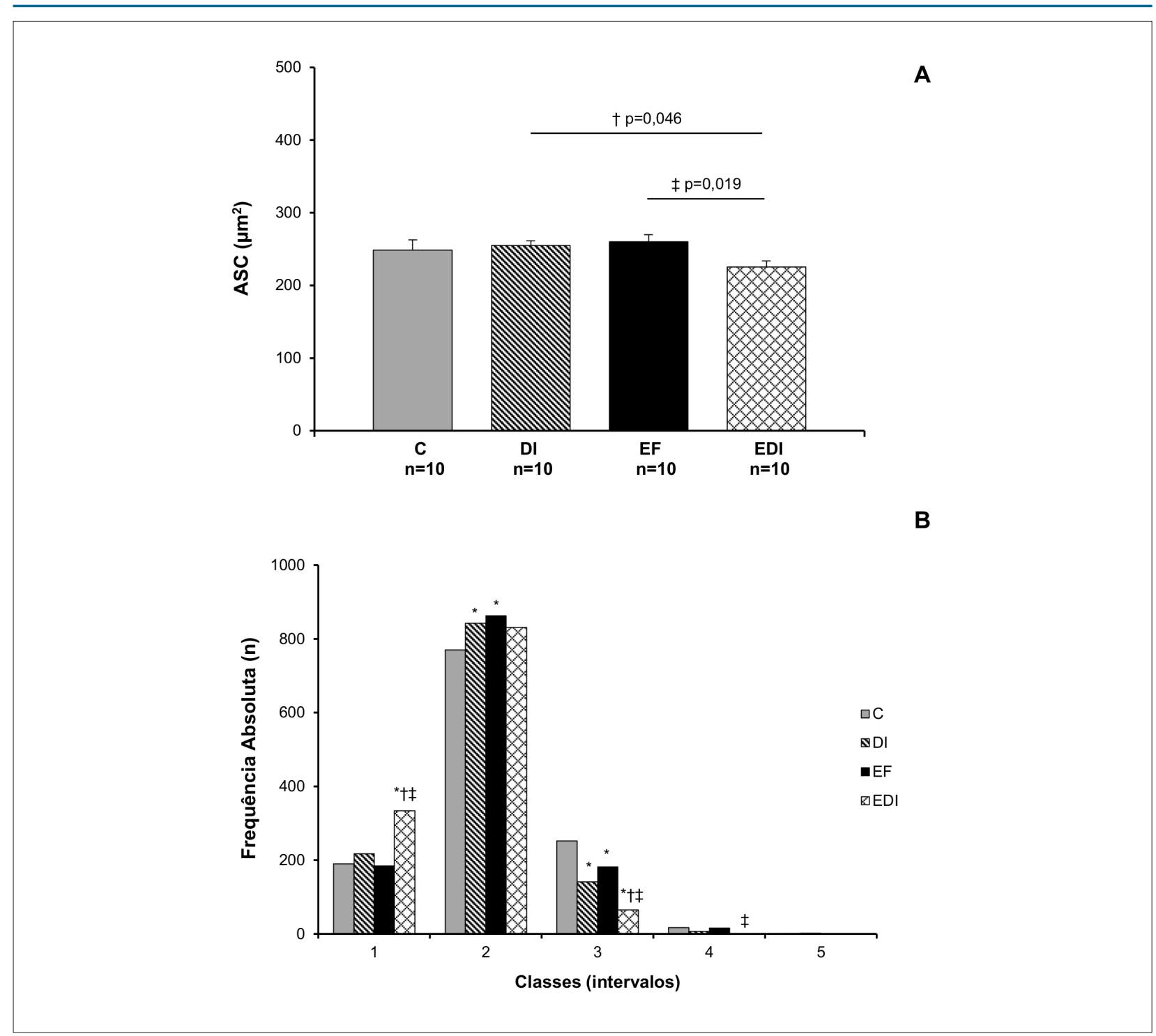

Figura 3 - (A) Área seccional transversa do cardiomiócito (ASC); $† p<0,05$ versus DI; $\ddagger p<0,05$ versus EF. Two-Way ANOVA e teste de Student-Newman-Keuls. (B) Distribuição de frequências de cardiomiócitos segundo intervalo de classes de ASC; Classes: 1 (52,7- 190,1 $\left.\mu \mathrm{m}^{2}\right), 2$ (190,1- 327,6 $\left.\mu \mathrm{m}^{2}\right), 3\left(327,6-465,0 \mu \mathrm{m}^{2}\right), 4$ $\left.(465,0\} 602,4 \mu \mathrm{m}^{2}\right)$ e $5\left(602,4-739,9 \mu \mathrm{m}^{2}\right) ;{ }^{*} p<0,05$ versus $C ; \uparrow p<0,05$ versus $D I ; \ddagger p<0,05$ versus EF. Teste de Goodman para contrastes dentro e entre populações multinomiais. C: Controle; DI: Dieta Intermitente; EF: Exercício Físico; EDI: Exercício Físico e Dieta Intermitente.

exercitados, mesmo apresentando aumento de fome, não exibiram significativo aumento da ingestão alimentar.

Segundo a hipótese inicial do presente estudo, o exercício físico em esteira rolante atenua prováveis desordens metabólicas e o processo de remodelação cardíaca decorrentes da restrição calórica intermitente. Além de reduzir a eficiência energética, a dieta intermitente modificou a tolerância glicêmica, o que pode estar associado com melhor sensibilidade à ação insulinêmica. A insulina tem propriedades lipogênicas sobre o tecido adiposo, ${ }^{33}$ fato que poderia explicar porque os grupos sob dieta intermitente, mesmo com massa corporal inferior, não apresentaram diferenças significativas de adiposidade corporal, quando comparados aos respectivos controles (Tabela 2). A insulina aumenta sua liberação quando há maior oferta de nutrientes, como no período pós-prandial. A melhora da sensibilidade à insulina pela adoção da dieta intermitente foi encontrada também em estudo recente com ratos. ${ }^{34}$

Por sua vez, a combinação entre dieta intermitente e exercício físico resultou em menor adiposidade corporal no EDI. O exercício físico promove adaptações e ajustes de natureza cardiorrespiratória, neural e hormonal.9,15,16 No contexto hormonal, a secreção dos hormônios é também alterada pelo exercício. No estudo de Evans et al., ${ }^{35}$ a prática de exercício físico aeróbio durante 12 meses resultou em melhora na sensibilidade à insulina, com diminuição de 19,4\% da área da curva de tolerância oral a glicose, em idosos. Portanto, a interação entre dieta e exercício pode ter potencializado os efeitos hormonais do metabolismo insulinêmico, como sustentado pelos achados nos grupos EF e EDI. 
Artigo Original

Tabela 3 - Expressão de proteínas MAPK do miocárdio, segundo grupo

\begin{tabular}{|c|c|c|c|c|}
\hline \multirow[b]{2}{*}{ Proteínas } & \multicolumn{4}{|c|}{ Grupo } \\
\hline & $\underset{n=6}{C}$ & $\begin{array}{c}D I \\
n=6\end{array}$ & $\begin{array}{c}E F \\
n=6\end{array}$ & $\begin{array}{l}\text { EDI } \\
n=6\end{array}$ \\
\hline p-ERK/ERK & $1,00 \pm 0,52$ & $1,42 \pm 1,59$ & $1,08 \pm 0,48$ & $1,23 \pm 0,78$ \\
\hline $\mathrm{p}$-ERK/GAPDH & $1,00 \pm 0,47$ & $1,18 \pm 1,19$ & $0,87 \pm 0,41$ & $0,86 \pm 0,32$ \\
\hline ERK/GAPDH & $1,00 \pm 0,10$ & $0,99 \pm 0,23$ & $0,91 \pm 0,16$ & $0.91 \pm 0,22$ \\
\hline p-JNK/JNK & $1,00 \pm 0,39$ & $1,00 \pm 0,35$ & $1,09 \pm 0,61$ & $1,03 \pm 0,40$ \\
\hline p-JNK/GAPDH & $1,00 \pm 0,13$ & $1,11 \pm 0,26$ & $1,10 \pm 0,35$ & $1,15 \pm 0,23$ \\
\hline JNK/GAPDH & $1,00 \pm 0,46$ & $1,04 \pm 0,42$ & $0,94 \pm 0,31$ & $0.96 \pm 0,32$ \\
\hline
\end{tabular}

Valores em média \pm desvio padrão; ERK: extracellular signal-regulated kinase; JNK: c-Jun N-terminal kinase; Two-Way ANOVA ( $p>0,05)$.

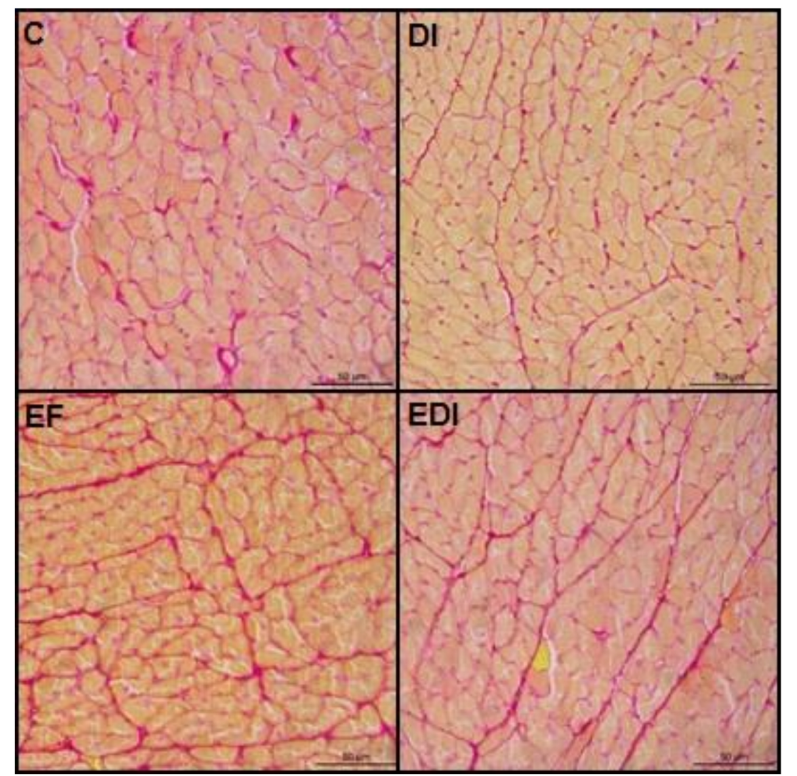

B

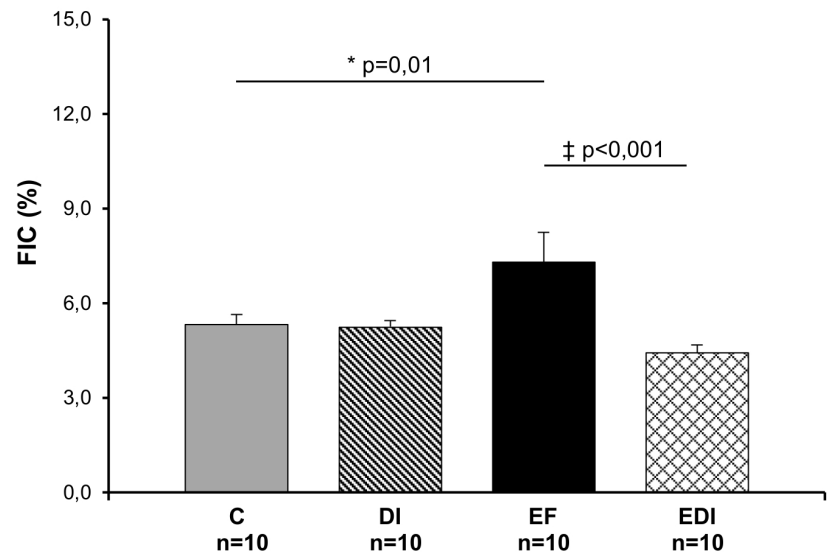

A 
Apesar das repercussões no metabolismo glicêmico, a dieta intermitente não interferiu na capacidade funcional. Para a determinação do limiar anaeróbio, a mensuração dos níveis de lactato é um dos parâmetros mais utilizados para estimar a capacidade aeróbia e tem se mostrado um eficaz índice de avaliação desta capacidade. ${ }^{22} \mathrm{O}$ limiar de lactato pode ser definido como a intensidade de exercício em que a concentração sanguínea de lactato tem um aumento abrupto. ${ }^{21,22}$ Nesse sentido, o protocolo de corrida resultou em melhor desempenho funcional dos grupos EF e EDI, o que foi sustentado por menores valores de lactato final (LacE), menor variação dos níveis de lactato e maiores valores de velocidade e distância percorrida durante o teste final. Portanto, pode-se afirmar que o exercício físico promoveu melhora da capacidade funcional, como já demonstrado. ${ }^{16}$

No aspecto cardiovascular, o exercício físico acarretou remodelação intersticial do miocárdio. Intrigantemente, a restrição intermitente promoveu retenção desses efeitos do exercício, o que foi sustentado por menores valores de morfometria macro e microscópica tecidual no EDI. Entre os fatores estimulantes da proliferação e estimulação do processo de remodelação miocárdica, incluem-se distúrbios nutricionais, angiotensina, aldosterona, endotelinas, citocinas inflamatórias e catecolaminas. ${ }^{36}$ Com a sobrecarga física prolongada, ocorrem alterações morfofuncionais no miocárdio, com o intuito de melhorar o desempenho do coração no bombeamento de sangue e na capacidade do sistema cardiovascular de fornecer oxigênio aos músculos recrutados durante o esforço. ${ }^{21,22}$ Entre essas adaptações do exercício, inclui-se a hipertrofia ventricular esquerda, desenvolvida para compensar a demanda hemodinâmica, e a fibrose intersticial. ${ }^{36,37}$ Nessa perspectiva, o aumento de colágeno intersticial encontrado no grupo EF, pode se configurar como indício do processo de remodelação ventricular fisiológica, ainda que os achados morfológicos não tenham confirmado a hipertrofia. Alguns fatores podem restringir a precisão da morfometria microscópica, incluindo-se variabilidade do ângulo de corte tecidual, estado contrátil heterogêneo das fibras cardíacas, entre outros. ${ }^{38}$ Tais condições podem ter contribuído para a não detecção de hipertrofia cardíaca derivada do exercício físico.

Por sua vez, restrição intermitente acarretou menores valores de morfologia macro e microscópica, sem afetar a expressão e ativação de proteínas ERK e JNK. As alterações fenotípicas carreadas por esses peptídeos envolvem síntese de proteínas e crescimento celular, com instalação de hipertrofia e fibrose intersticial, as quais podem ser associadas ao processo de remodelação miocárdica. ${ }^{13,14}$ Além disso, a ativação de MAPK é também subordinada à ação de fatores de crescimento, como hormônio do crescimento e insulina, ${ }^{24,33}$ os quais têm sua secreção regulada pelo comportamento nutricional. Apesar disso, não foi possível verificar a associação entre dieta intermitente e alteração na expressão de MAPK. Em estudo prévio. ${ }^{39}$ a dieta intermitente reduziu a hipertrofia cardíaca e a dilatação ventricular em ratos infartados, embora não tenha alterado a expressão gênica de peptídeos fetais.

Com o presente estudo, tem-se, portanto, a comprovação de que a remodelação cardíaca derivada do exercício físico foi amenizada pela dieta intermitente. Entretanto, não é possível afirmar se esse potencial profilático da intervenção dietética pode ser utilizado na reversão de processos patológicos, como na hipertensão arterial e infarto agudo do miocárdio. Da mesma forma, o impacto de outros modelos experimentais, incluindo-se 25 e 50\% de restrição calórica ${ }^{5,7-9}$ deve ser melhor estudado em futuras investigações. Tais fatos trazem limitações em termos de repercussões clínicas para os resultados da presente investigação.

\section{Conclusão}

A combinação de dieta intermitente e exercício físico se associa com maior tolerância glicêmica. O exercício físico, isoladamente, causa remodelação intersticial do miocárdio, que é atenuada pela intervenção com dieta intermitente.

\section{Contribuição dos Autores}

Concepção e desenho da pesquisa: Basilio PG, OliveiraJunior SA; Obtenção de dados: Basilio PG, Oliveira APC, Castro ACF, Carvalho MR, Zagatto AM, Martinez PF, Ota GE; Análise e interpretação dos dados: Zagatto AM, Martinez PF, Okoshi MP, Okoshi K, Reis FA, Oliveira-Junior SA; Análise estatística: Okoshi K, Oliveira-Junior SA; Obtenção de financiamento: Oliveira-Junior SA; Redação do manuscrito: Basilio PG, Oliveira-Junior SA; Revisão crítica do manuscrito quanto ao conteúdo intelectual importante: Zagatto AM, Martinez PF, Okoshi MP, Okoshi K, Reis FA.

\section{Potencial Conflito de Interesses}

Declaro não haver conflito de interesses pertinentes.

\section{Fontes de Financiamento}

O presente estudo teve apoio financeiro da Coordenação de Aperfeiçoamento de Pessoal de Nível Superior - Brasil (CAPES) - Código de Financiamento 001, Conselho Nacional de Desenvolvimento Científico e Tecnológico (CNPq), Fundação de Apoio ao Desenvolvimento do Ensino, Ciência e Tecnologia do Estado do Mato Grosso do Sul (FUNDECT) e Universidade Federal de Mato Grosso do Sul (UFMS).

\section{Vinculação Acadêmica}

Este artigo é relacionado à dissertação de Mestrado de Priscilla Gois Basílio pela Universidade Federal do Mato Grosso do Sul. 


\section{Referências}

1. Weindruch $\mathrm{R}$. The retardation of aging by caloric restriction: studies in rodents and primates. Toxicol Pathol. 1996;24(6):742-5.

2. Anderson RM, Shanmuganayagam D, Weindruch R. Caloric restriction and aging: studies in mice and monkeys. Toxicol Pathol. 2009;37(1):47-51.

3. Fontana L, Partridge L, Longo VD. Extending healthy life span - from yeast to humans. Science. 2010;328(5976):321-6.

4. Colman RJ, Beasley TM, Allison DB, Weindruch R. Attenuation of sarcopenia by dietary restriction in rhesus monkeys. J Gerontol A Biol Sci Med Sci. 2008;63(6):556-9.

5. Okoshi MP, Okoshi K, Dal Pai V, Dal Pai-Silva M, Matsubara LS, Cicogna AC. Mechanical, biochemical, and morphological changes in the heart from chronic food-restricted rats. Can J Physiol Pharmacol. 2001;79(9):754-60.

6. Guo Z, Mitchell-Raymundo F, Yang H, Ikeno Y, Nelson J, Diaz V, et al. Dietary restriction reduces atherosclerosis and oxidative stress in the aorta of apolipoprotein E-deficient mice. Mech Ageing Dev. 2002;123(8):1121-31.

7. Gut AL, Okoshi MP, Padovani CR, Aragon FF, Cicogna AC. Myocardial dysfunction induced by food restriction is related to calcium cycling and beta-adrenergic systems changes. Nutr Res. 2003;23(7):911-9.

8. Sugizaki MM, Carvalho RF, Aragon FF, Padovani CR, Okoshi K, Okoshi $\mathrm{MP}$, et al. Myocardial dysfunction induced by food restriction is related to morphological damage in normotensive middle-aged rats. J Biomed Sci. 2005;12(4):641-9.

9. Sugizaki MM, Pai-Silva MD, Carvalho RF, Padovani CR, Bruno A, Nascimento $\mathrm{AF}$, et al. Exercise training increases myocardial inotropic response in food restricted rats. Int J Cardiol. 2006; 112(2):191-201.

10. Cicogna AC, Padovani CR, Georgette JC, Aragon FF, Okoshi MP. Effects of protein-calorie restriction on mechanical function of hypertrophied cardiac muscle. Arq Bras Cardiol. 1999; 72(4):431-40.

11. Pinotti MF, Leopoldo AS, Pai-Silva MD, Sugizaki MM, Nascimento AF, Leopoldo APL, et al. A comparative study of myocardial function and morphology during fasting/refeeding and food restriction in rats. Cardiovasc Pathol. 2010;19(5):e175-82.

12. Ahmet I, Tae HJ, De Cabo R, Lakatta EG, Talan MI. Effects of calorie restriction on cardioprotection and cardiovascular health. J Mol Cell Cardiol. 2011;51(2):263-71.

13. Wang Y. Mitogen-activated protein kinases in heart development and diseases. Circulation. 2007;116(12):1413-23.

14. Rose BA, Force T, Wang Y. Mitogen-activated protein kinase signaling in the heart: angels versus demons in a heart-breaking tale. Physiol Rev. 2010;90(4):1507-46.

15. Sugizaki MM, Leopoldo AP, Conde SJ, Campos DS, Damato R, Leopoldo AS. Upregulation of mRNA myocardium calcium handling in rats submitted to exercise and food restriction. Arq Bras Cardiol. 2011;97(1):46-52.

16. Pagan LU, Damatto RL, Cezar MDM, Bonomo C, Campos DHS, Gomes MJ, et al. Long-term low intensity physical exercise attenuates heart failure development in aging spontaneously hypertensive rats. Cell Physiol Biochem. 2015;36(1):61-74

17. Palmer BM, Thayer AM, Snyder SM, Moore RL. Shortening and [Ca2+] dynamics of left ventricular myocytes isolated from exercise-trained rats. J Appl Physiol (1985). 1998;85(6):2159-68.

18. Broderick TL, Driedzic WR, Gillis M, Jacob J, Belke T. Effects of chronic food restriction and exercise training on the recovery of cardiac function following ischemia. J Gerontol A Biol Sci Med Sci. 2001;56(1):B33-7.

19. Weiner RB, Baggish AL. Exercise-induced cardiac remodeling. Prog Cardiovasc Dis. 2012;54(5):380-6.

20. Emter CA, McCune SA, Sparagna GC, Radin MJ, Moore RL. Lowintensity exercise training delays onset of decompensated heart failure in spontaneously hypertensive heart failure rats. Am J Physiol Heart Circ Physiol. 2005;289(5):H2030-8.
21. Carvalho JF, Masuda MO, Pompeu FAMS. Method for diagnosis and contro of aerobic training in rats based on lactate threshold. Comp Biochem Physiol A Mol Integr Physiol. 2005;140(4):409-13.

22. Mendes OC, Sugizaki MM, Campos DS, Damatto RL, Leopoldo AS Lima-Leopoldo AP, et al. Exercise tolerance in rats with aortic stenosis and ventricular diastolic and/or systolic dysfunction. Arq Bras Cardiol. $2013 ; 100(1): 44-51$

23. Oliveira Jr SA, Okoshi K, Lima-Leopoldo AP, Leopoldo AS, Campos DH Martinez PF, et al. Nutritional and cardiovascular profiles of normotensive and hypertensive rats kept on a high fat diet. Arq Bras Cardiol. 2009;93(5):526-33.

24. Oliveira-Junior SA, Martinez PF, Guizoni DM, Campos DH, Fernandes T, Oliveira EM, et al. AT1 receptor blockade attenuates insulin resistance and myocardial remodeling in rats with diet-induced obesity. Plos One. 2014;9(1):e86447.

25. Martins F, Campos DHS, Pagan LU, Martinez PF, Okoshi K, Okoshi MP, et al. High-fat diet promotes cardiac remodeling in an experimental model of obesity. Arq Bras Cardiol. 2015;105(5):479-86.

26. Martinez PF, Okoshi K, Zornoff LAM, Carvalho RF, Oliveira Junior SA, Lima ARR, et al. Chronic heart failure-induced skeletal muscle atrophy, necrosis, and changes in myogenic regulatory factors. Med Sci Monit. 2010;16(12):BR374-83

27. Bradford MM. A rapid and sensitive method for the quantitation of microgram quantities of protein utilizing the principle of protein-dye binding. Anal Biochem. 1976;72(1-2):248-54.

28. Norman GR. Biostatistics - The bare essentials, Mosby, St. Louis, 1998.

29. Goodman LA. On the multivariate analysis of three dichotomous variables. Am J Sociol. 1965;71(3):290-301.

30. Chausse B, Solon C, Caldeira da Silva CC, Masselli Dos Reis IG, ManchadoGobatto FB, Gobatto CA, et al. Intermittent fasting induces hypothalamic modifications resulting in low feeding efficiency, low body mass and overeating. Endocrinology. 2014;155(7):2456-66.

31. Chausse B, Vieira-Lara MA, Sanchez AB, Medeiros MH, Kowaltowski AJ Intermittent fasting results in tissue - specific changes in bioenergetics and redox state. PLoS One. 2015 Mar 6;10(3):e0120413.

32. Dorighello GG, Rovani JC, Luhman CJ, Paim BA, Raposo HF, Vercesi AE, et al. Food restriction by intermittent fasting induces diabetes and obesity and aggravates. Br J Nutr. 2014;111(6):979-86.

33. Bhutani S, Klempel MC, Kroeger CM, Aggour E, Calvo Y, Trepanowski JF, et al. Effect of exercising while fasting on eating behaviors and food intake. Int Soc Sports Nutr. 2013;10(1):50.

34. Saltiel AR, Kahn CR. Insulin signalling and the regulation of glucose and lipid metabolism. Nature. 2001;414(6865):799-806.

35. Baumeier C, Kaiser D, Heeren J, Scheja L, John C, Weise C, et al. Caloric restriction and intermittent fasting alter hepatic lipid droplet proteome and diacylglycerol species and prevent diabetes in NZO mice. Biochim Biophys Acta. 2015;1851(5):566-76.

36. Azevedo PS, Polegato BF, Minicucci MF, Paiva SA, Zornoff LA. Cardiac remodeling: concepts, clinical impact, pathophysiological mechanisms and pharmacologic treatment. Arq Bras Cardiol. 2016;106(1):62-9.

37. Souza FR, Resende ES, Lopes L, Gonçalves A, Chagas R, Fidale T, et al Hypertrophic response of the association of thyroid hormone and exercise in the heart of rats. Arq Bras Cardiol. 2014;102(2):187-90.

38. Gerdes AM. Cardiac myocyte remodeling in hypertrophy and progression to failure. J Card Fail. 2002; 8(6):264-8.

39. Okoshi K, Cezar MDM, Polin MAM, Paladino JrJR, Martinez PF, Oliveira J $\mathrm{SA}$, et al. Influence of intermittent fasting on myocardial infarction-induced cardiac remodeling. BMC Cardiovasc Disord. 2019;19:126. 